\title{
Brain Functional Network Improved by Magnetic Stimulation at Acupoints during Mental Fatigue
}

\author{
Shuo Yang, Na Ai, Yanyun Qiao, Lei Wang, Hongli Yu, Guizhi Xu \\ Province-Ministry Joint Key Laboratory of Electromagnetic Field and Electrical Apparatus Reliability, Hebei University of Technology, \\ Tianjin, China \\ Email: 13820527919@163.com
}

How to cite this paper: Yang, S., Ai, N., Qiao, Y.Y., Wang, L., Yu, H.L. and Xu, G.Z. (2016) Brain Functional Network Improved by Magnetic Stimulation at Acupoints during Mental Fatigue. J. Biomedical Science and Engineering, 9, 65-70.

http://dx.doi.org/10.4236/jbise.2016.910B009

Received: July 14, 2016

Accepted: September 20, 2016

Published: September 23, 2016

\begin{abstract}
To investigate the effects of magnetic stimulation at acupoints on brain functional network during mental fatigue, magnetic stimulation was applied to stimulate SHENMEN (HT7), HEGU (LI4) and LAOGONG (PC8) acupoint in this paper. The brain functional networks of normal state, mental fatigue state and stimulated state were constructed and the characteristic parameters were comparatively studied based on the complex network theory. The results showed that the connection of the network was enhanced by stimulating the HT7, LI4 and PC8 acupoint. In conclusion, magnetic stimulation at acupoints can effectively relieve mental fatigue.
\end{abstract}

\section{Keywords}

Magnetic Stimulation, Acupoint, Electroencephalograph (EEG), Mental Fatigue, Brain Functional Network

\section{Introduction}

Mental fatigue has been defined as a state resulting from the prolonged periods of cognitive activity [1]. Mental fatigue impairs cognitive performances [2], and it is a major human factor in the safety of transportation systems [3]. It is extremely necessary to detect and relieve mental fatigue. Acupuncture can relieve physical and mental fatigue in patients with Chronic Fatigue Syndrome (CFS) [4] [5], but its operation is difficult to quantify [6]. Magnetic stimulation, as a novel stimulus, demonstrates a promising application prospect in clinical including regulation of human neural function and disease treatment. In this paper, magnetic stimulation at acupoints was used. The brain functional networks of the three states were constructed and the changes of brain connection were comparatively analyzed from the perspective of complex network [7]. 


\section{Materials and Methods}

\subsection{Subjects}

Fourteen healthy male undergraduates 20 to 23 years old were selected, with consent obtained before the study. They were all right handed. They had no major brain disease, mental disorders and organic disease. Caffeine, tea, alcohol, smoking were prohibited and they were required not to undertake strenuous exercise before the experiment.

\subsection{Selection of Stimulation Points}

The stimulated acupoint was selected following the acupuncture map and the International Standard Nomenclature of Acupuncture. The SHENMEN (HT7), HEGU (LI4) and LAOGONG (PC8) acupoint were chosen in the magnetic stimulation experiment. They were commonly used to relieve mental fatigue and CFS with acupuncture [8]. Figure 1 shows the locations of HT7, LI4 and PC8 acupoint.

\subsection{Magnetic Stimulation Experimental Protocol}

The trials were carried out in a quiet and air-conditioned room maintained at around $22^{\circ} \mathrm{C}$. The subjects were asked to learn scientific papers in English from 8:30 a.m. to 12:30 p.m. in the cognitive task. The sequence of magnetic stimulation was carried out by a Magstim Rapid2 magnetic stimulator and an 8-shaped coil (9925-00) with a peak magnetic field of $2.2 \mathrm{~T}$. The magnetic stimulation frequency was $1 \mathrm{~Hz}$ and the intensity of stimulation is $80 \%$ of the input power. The center of the 8 -shaped coil is right over the acupoint. The stimulation time of each acupoint is $90 \mathrm{~s}$, and the rest time between the adjacent acupoints is $60 \mathrm{~s}$.

\subsection{EEG Recording and Pre-Processing}

64-channelEEG recording was through the Neuro Scan EEG recording system for two minutes with the M1 and M2 electrodes set as reference electrodes. The sampling rate was $1000 \mathrm{~Hz}$. Electrode impedances was kept below $5 \mathrm{k} \Omega$. At the same time, subjects need to fill in the Karolinska Sleepiness Scale (KSS) in normal state, mental fatigue state and stimulated state. The principle of the whole experiment was shown in Figure 2.
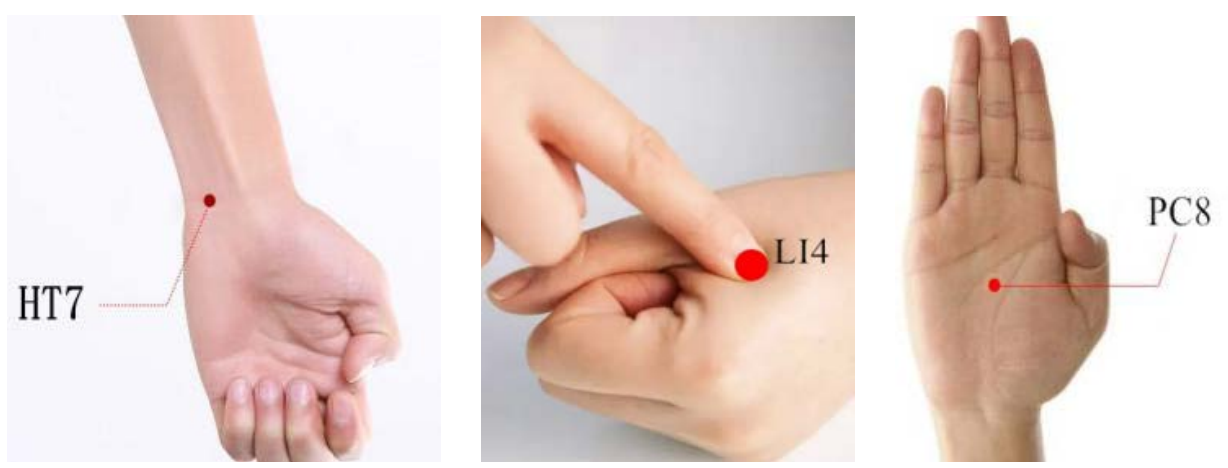

Figure 1. Locations of HT7, LI4 and PC8 acupoint. 


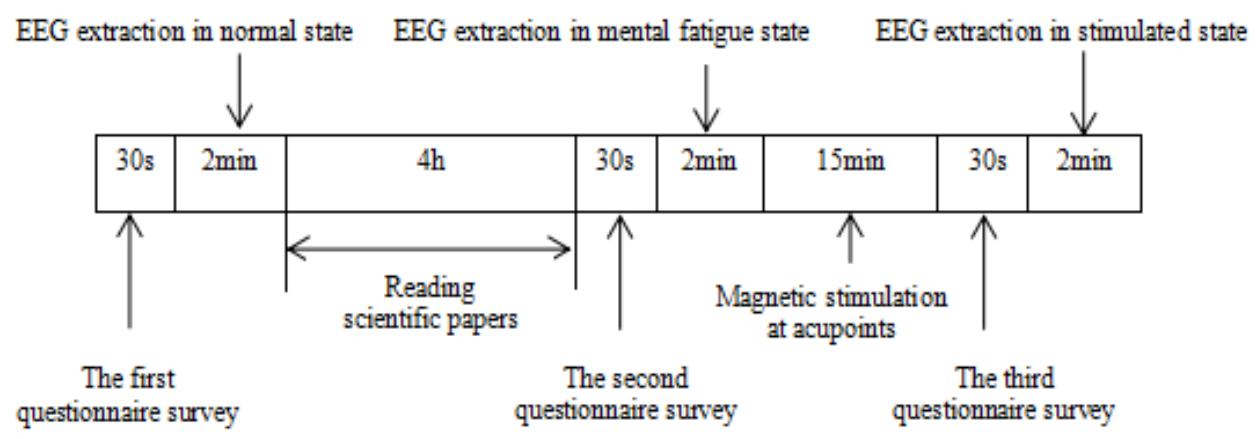

Figure 2. The principle of the experiment.

EEG data must be processed including DC offset correction, artifact rejection, data filtering, epoch, baseline correction, and linear-detread.

\subsection{The Construction and Statistical Analysis of Brain Functional Network}

The linear method of mutual correlation was used to estimate quantitatively correlation between different channels of EEG signals. For threshold selection, we used network density (S) as an indicator of the threshold [9] and to maintain the network connectivity, the average degree $(\mathrm{K})$ should meet the following conditions: $N \geq K \geq \ln (\mathrm{N}) \gg 0$. ( N is the total number of channels).

$$
\begin{aligned}
& S=\frac{1}{N(N-1)} \sum_{i=1}^{N} K_{i} \\
& K=\frac{1}{N} \sum_{i=1}^{N} K_{i}
\end{aligned}
$$

where $K_{i}$ is the degree of node $i$.

Then the corresponding brain functional networks were constructed and the characteristic parameters, including average degree, average clustering coefficient, network density and average path length, were analyzed with paired sample $t$ test for normal state, mental fatigue state and stimulated state. The scores of KSS were also studied by the same method. They have statistical significance if $\mathrm{p}<0.05$.

\section{Results}

\subsection{Statistical Analysis of Questionnaire}

The KSS scores showed that the score in mental fatigue state was higher than that of normal state, and became lower after magnetic stimulation (normal state: $3.50 \pm 1.22$, mental fatigue state: $6.29 \pm 1.14$, stimulated state: $4.29 \pm 1.44$ ). These results indicate that the sustained cognitive task significantly increased subjective feeling of mental fatigue, and mental fatigue was effectively relieved by magnetic stimulation at acupoints (between normal state and mental fatigue state: $t=-9.92, p<0.05$, between mental fatigue state and stimulated state: $\mathrm{t}=4.50, \mathrm{p}<0.05)$. 


\subsection{Brain Functional Network Construction}

We set the threshold value to 0.68 according to the threshold selection strategy ( $\mathrm{K} \geq$ $2 \ln (\mathrm{N}) \approx 8, \mathrm{~S}<50 \%$ ), got the correlation coefficients matrix of one subject for three states (Figure 3) and constructed the corresponding brain functional networks (Figure 4). By the way, the characteristic parameters of the networks were analyzed for normal state, mental fatigue state and stimulated state (Table 1).

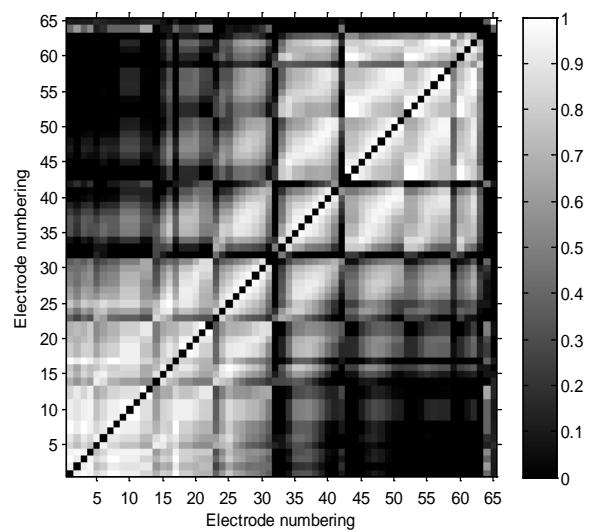

(a)

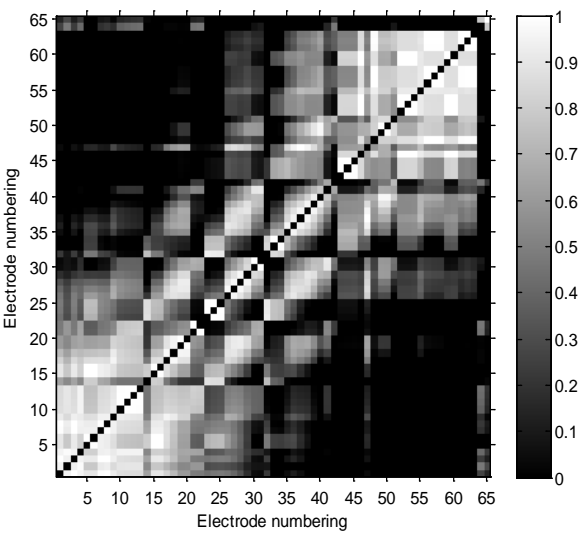

(b)

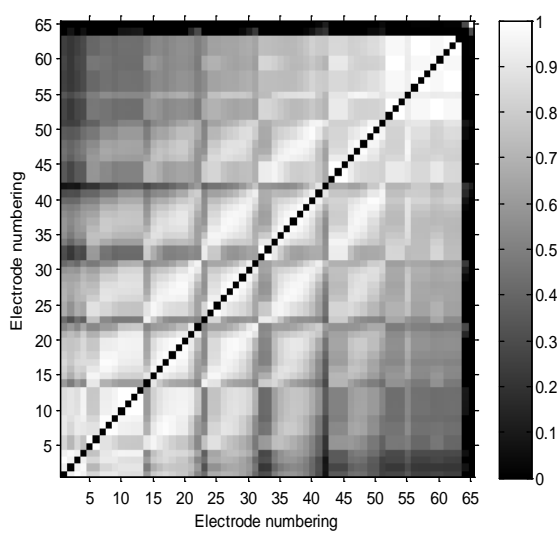

(c)

Figure 3. The mutual correlation coefficients matrices for three states. (a) Normal state; (b) Mental fatigue state; (c) Stimulated state.

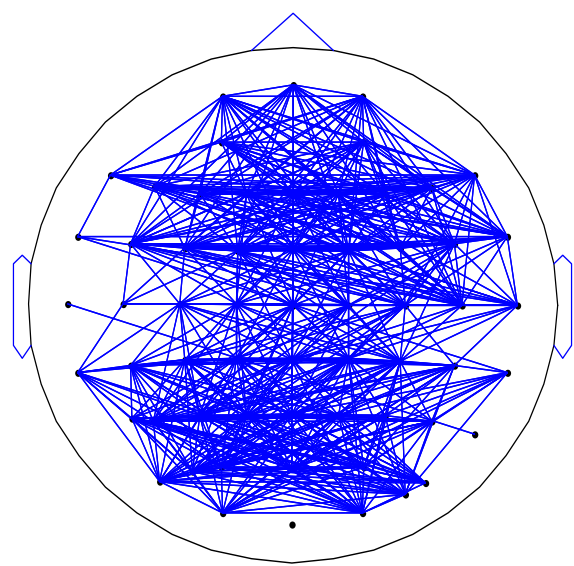

(a)

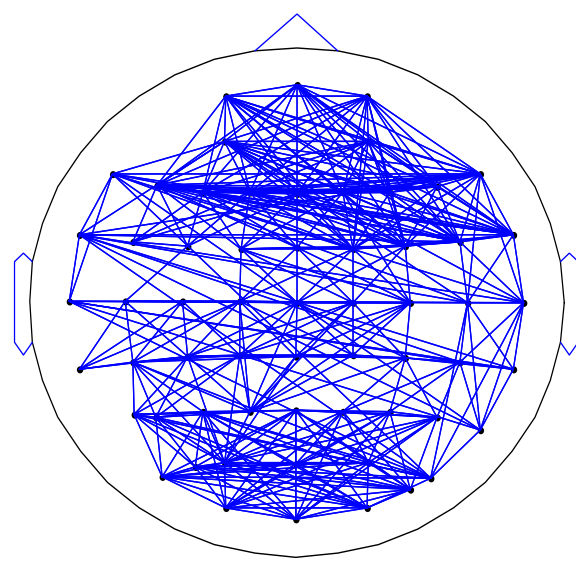

(b)

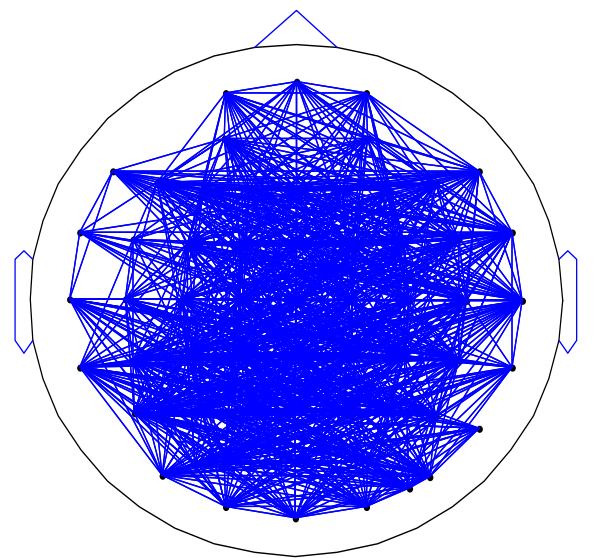

(c)

Figure 4. Brain functional networks for three states. (a) Normal state; (b) Mental fatigue state; (c) Stimulated state.

Table 1. The characteristic parameters of network topology of three states $\left(\begin{array}{l}\bar{X} \\ \pm s\end{array}\right)$.

\begin{tabular}{ccccc}
\hline Parameters States & Average Degree & Average Clustering Coefficient & Network Density & Average Path Length \\
\hline Normal state & $19.30 \pm 5.52$ & $0.76 \pm 0.05$ & $0.30 \pm 0.09$ & $2.22 \pm 0.35$ \\
Mental fatigue state & $16.11 \pm 3.78^{*}$ & $0.72 \pm 0.07^{*}$ & $0.25 \pm 0.06^{*}$ & $2.43 \pm 0.39^{*}$ \\
Stimulated state & $21.26 \pm 8.14^{* *}$ & $0.76 \pm 0.05^{* *}$ & $0.33 \pm 0.13^{* *}$ & $2.00 \pm 0.42^{* *}$ \\
\hline
\end{tabular}

Note: ${ }^{*}$ : significant difference between normal state and mental fatigue state $(\mathrm{p}<0.05) .{ }^{* *}$ : significant difference between mental fatigue state and stimulated state $(\mathrm{p}<$ $0.05)$. 
These results indicate that mental fatigue was successfully induced by the sustained cognitive task ( $\mathrm{p}<0.05)$, and significantly relieved by magnetic stimulation $(\mathrm{p}<0.05)$. The average degree, average clustering coefficient and network density of the brain functional network were reduced, while the average pathlength was increased during mental fatigue. However, the average degree, average clustering coefficient and network density were increased by stimulating the HT7, LI4 and PC8 acupoint, while the average pathlength was reduced. The results show that the connectivity of the brain and the efficiency of information transmission are both decreased during mental fatigue. While the complexity of network is significantly enhanced and the information transfer among brain regions is more efficiently due to magnetic stimulation. It has also revealed that the correlation of the network is higher than that of the normal state.

\section{Conclusions}

This study is to investigate the effects of magnetic stimulation at acupoints during mental fatigue from the perspective of brain functional network. The linear method of mutual correlation in the time domain is used to study the 64-channel EEG data in three states (normal state, mental fatigue state and stimulated state), and complex network theory is introduced to construct and analyze the brain functional network.

By comparing the three states (normal state, mental fatigue state and stimulated state) of networks, the results indicate that the average degree, average clustering coefficient and network density were reduced during mental fatigue, while the average path length was increased. It means that the connection of the brain functional network and the efficiency of information transmission are both decreased during mental fatigue. After magnetic stimulation at acupoints, the topology is changed, the connection of the network is enhanced, the efficiency of information transmission is improved and the small-world property is strengthened by stimulating the HT7, LI4 and PC8 acupoint. Moreover, we can see that the complexity of brain functional network and the correlation of brain regions after stimulated state are significantly increased than that of normal state. In a conclusion, the results indicate that mental fatigue state can be effectively alleviated by magnetic stimulation at HT7, LI4 and PC8 acupoint.

\section{Acknowledgements}

This study was supported by the National Natural Science Foundation of China, No. 31300818, No. 31400844 and the Foundation of Hebei Province Department of Education, No. QN2016097.

\section{References}

[1] Garnier, M. and Delamare, V. (1986) Dictionnaire des termes techniques de médecine, Maloine, Paris.

[2] Boksem, M.A.S., Meijman, T.F. and Lorist, M.M. (2005) Effects of Mentalfatigue on Attention: An ERP Study. Brain Res Cogn Brain Res, 25, 107-116.

http://dx.doi.org/10.1016/j.cogbrainres.2005.04.011

[3] Akerstedt, T. (2000) Consensus Statement: Fatigue and Accidents in Transport Operations. 
Journal of Sleep Research, 9, 395. http://dx.doi.org/10.1046/j.1365-2869.2000.00228.x

[4] Lu, C., Yang, X.J. and Hu, J. (2014) Randomized Controlled Clinical Trials of Acupuncture and Moxibustion Treatment of Chronic Fatigue Syndrome Patients. Acupuncture Research, 39, 313-317.

[5] Wang, J.J., Song, Y.J., Wu, Z.C., et al. (2009) Randomized Controlled Clinical Trials of Acupuncture Treatment of Chronic Fatigue Syndrome. Acupuncture Research, 34, 120124.

[6] Na, B.J., Jahng, G.H., Park, S.U., et al. (2009) An fMRI Study of Neuronal Specificity of an Acupoint: Electroacupuncture Stimulation of Yanglingquan (GB34) and Its Sham Point. Neurosci Let, 464, 1-5. http://dx.doi.org/10.1016/j.neulet.2009.08.009

[7] Liang, X., Wang, J.H. and He, Y. (2010) Human Connectome: Structural and Functional Brain Networks. Chinese Science Bulletin, 55, 1565-1583.

http://dx.doi.org/10.1360/972009-2150

[8] Ng, S.M. and Yiu, Y.M. (2010) Acupuncture for Chronic Fatigue Syndrome: A Randomized, Sham-Controlled Trial with Single-Blinded Design. Alternative Therapies in Health and Medicine, 19, 21-26.

[9] Achard, S. and Bullmore, E. (2007) Efficiency and Cost of Economical Brain Functional Networks. Plos Computational Biology, 3, 174-183.

http://dx.doi.org/10.1371/journal.pcbi.0030017

\section{Submit or recommend next manuscript to SCIRP and we will provide best service for you:}

Accepting pre-submission inquiries through Email, Facebook, LinkedIn, Twitter, etc.

A wide selection of journals (inclusive of 9 subjects, more than 200 journals)

Providing 24-hour high-quality service

User-friendly online submission system

Fair and swift peer-review system

Efficient typesetting and proofreading procedure

Display of the result of downloads and visits, as well as the number of cited articles

Maximum dissemination of your research work

Submit your manuscript at: http://papersubmission.scirp.org/

Or contact jbise@scirp.org 